\title{
A Case of Spondylodiscitis due to Bacteroides Fragilis in Febrile Patient With Past Thoracic Vertebral Trauma
}

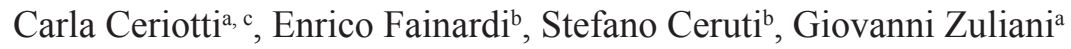

\begin{abstract}
Spondylodiscitis caused by Bacteroides fragilis in an uncommon finding. We report a case of spondylodiscitis due to Bacteroides fragilis in a febrile young patient with history of previous trauma to the back. Sub-acute appendicitis was considered the portal of entry of infection. The patient was febrile but did not complain spinal pain. 2- $\left[{ }^{18} \mathrm{~F}\right]$ fluoro-2-deoxy-D-glucose (FDG) positron emission tomography (PET) was very useful in the diagnosis of this pathology.
\end{abstract}

Keywords: Spondylodiscitis; Bacteroides fragilis; Febrile patient; Thoracic vertebral trauma

\section{Introduction}

Spondylodiscitis due to Bacteroides fragilis is an unfrequent finding that often results from hematogenous spread from a digestive portal of entry. No previous case reports have described spondylodiscitis due to Bacteroides fragilis that occurred after a spine trauma. We assume that spine trauma could represent a "locus minoris resistentiae" facilitating the initiation of a new focus of infection for Bacteroides fragilis from a primary intestinal infective focus.

\footnotetext{
Manuscript accepted for publication July 5, 2013

${ }^{a}$ Department of Medical Sciences, Section of Internal Medicine, Gerontology and Clinical Nutrition, S. Anna University Hospital, Ferrara, Italy

${ }^{\mathrm{b}}$ Neuroradiology Unit, Department of Neuroscience and Rehabilitation, S. Anna University Hospital, Ferrara, Italy

${ }^{\mathrm{c}}$ Corresponding author: Carla Ceriotti, Department of Medical

Sciences, Section of Internal Medicine, Gerontology and Clinical Nutrition, S. Anna University Hospital, Via Aldo Moro, 44124 Cona, Ferrara, Italy. Email: carlaceriotti@libero.it
}

doi: http://dx.doi.org/10.4021/jmc1391w

\section{Case Report}

A 41-year-old white Caucasian man was admitted to our Operative Unit because of abdominal pain and fever. Past medical history revealed bilateral nephrolithiasis and depressive syndrome. One year before he had experienced a thoracic trauma, secondary to car crash, without any neurological consequences. His medications included an antidepressive drug (venlafaxine) and benzodiazepines.

On admission the patient presented a temperature of $38.8^{\circ} \mathrm{C}$ and complained colicky abdominal pain. A thorough physical examination was performed. Abdominal examination revealed diffuse tenderness without signs of peritoneal irritation; Murphy's sign, renal and appendicular punches were negative. Heart, neurological, and thoracic examinations were unremarkable. On laboratory tests, the cell count demonstrated 12,470 white blood cells per cubic millimeter, with $70 \%$ neutrophils. C-reactive protein (PCR) was $21.6 \mathrm{mg} / \mathrm{dL}$. Hepatic, renal, coagulative function, and urinalysis were within normal limits. Blood and urine cultures were negative. Abdominal X-ray, chest radiography, and abdominal ultrasounds showed no abnormalities. The abdominal CT scan demonstrated sub-acute appendicitis. Surgical treatment was not indicated and an empirical antibiotic therapy with I.V. levofloxacin and antipiretic drug (paracetamol) and was started. After 7 days, the patient presented a complete remission of the symptoms; a control TC scan of the abdomen showed the regression of appendicular inflammation; nevertheless, within a few days fever accompanied by shivering recurred. Because of the persistent febrile status of unknown origin, blood culture specimens were further collected and a $2-\left[{ }^{18} \mathrm{~F}\right]$ fluoro2-deoxy-D-glucose (FDG) positron emission tomography (PET) was requested. Contrast tomography (CT) attenuation images were used for PET correction (PET/TC). PET/ TC revealed an intense FDG uptake in the intervertebral disc between thoracic vertebra 6 and 7; no abnormal FDG uptake was recognized in other areas and diagnosis of discitis was made (Fig. 1). T2-weighted magnetic resonance imaging (MRI) revealed a hyperintense signal in T6 and T7 vertebral bodies and in the corresponding intervertebral disc 


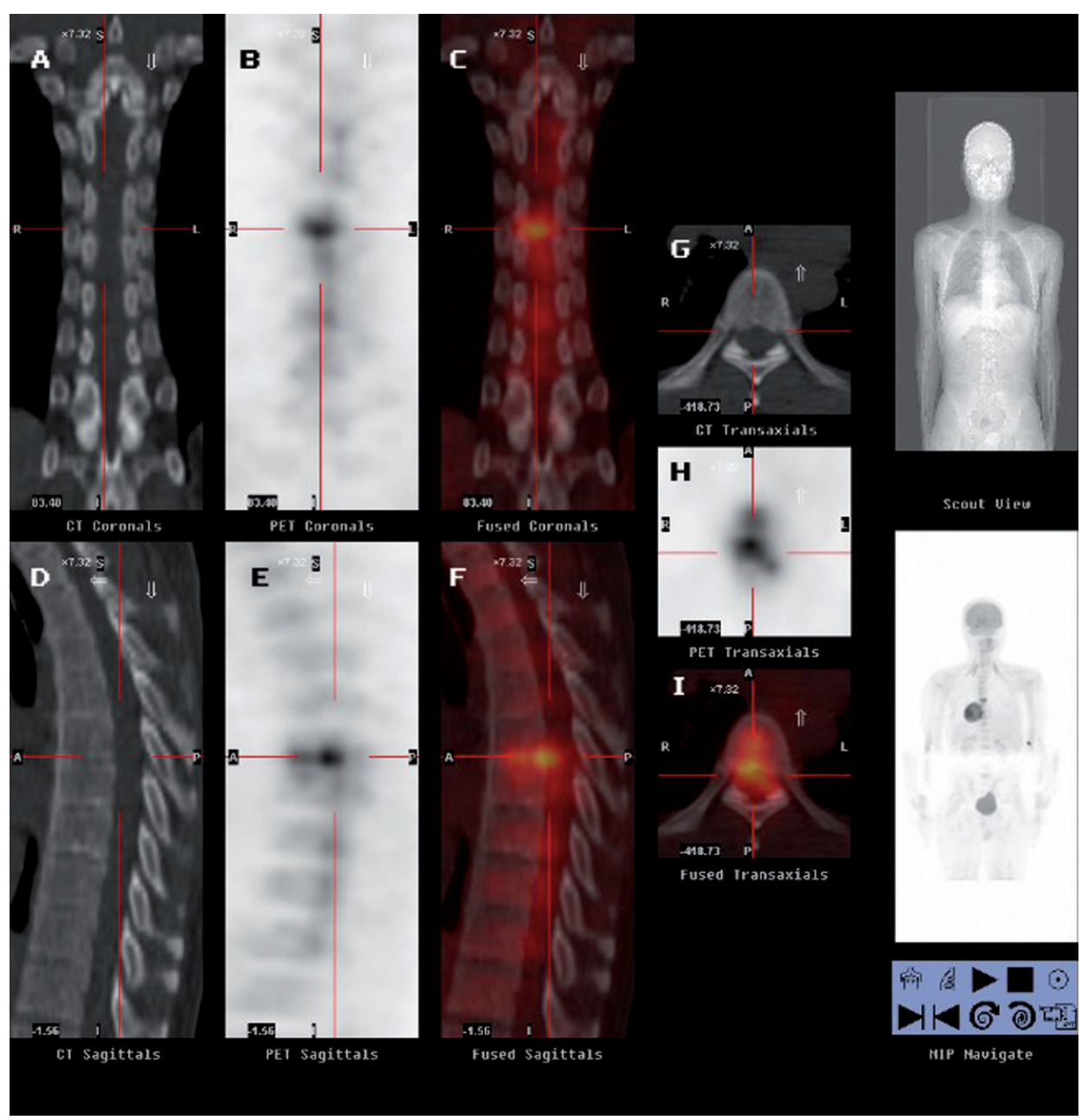

Figure 1. Images of patient's thoracic spine demonstrating the presence of inflammatory process in the intervertebral disc between T6 and T7. CT (A), PET (B), and fused (C) coronal view; CT (D), PET (E), and fused (F) sagittal view; CT $(G)$, PET $(H)$, and fused $(I)$ transaxial view.

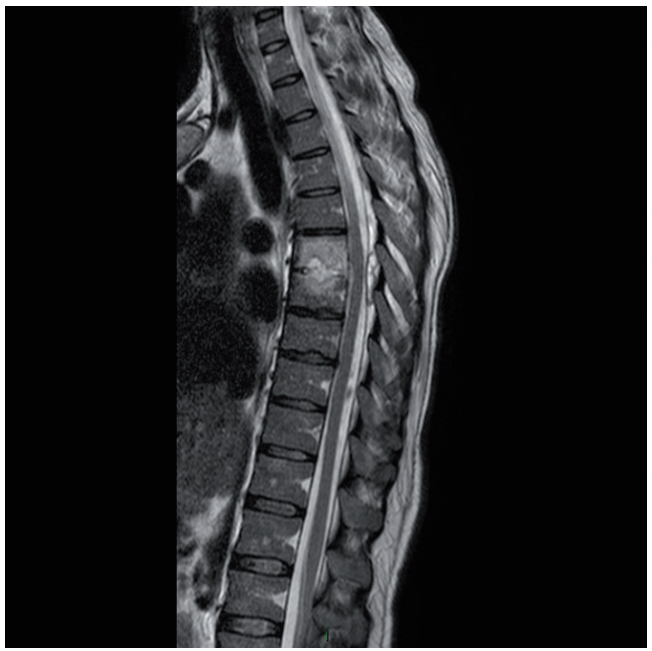

Figure 2. T2-weighted sagittal image of patient's thoracic spine showing hyperintense signal in T6 and T7 vertebral bodies and in the corresponding intervertebral disc.

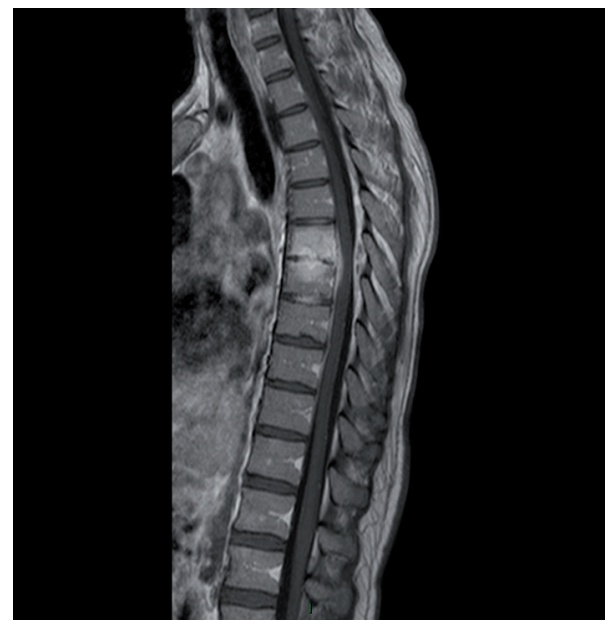

Figure 3. Post-gadolinium T1-weighted MRI sagittal image demonstrating avid enhancement of the lesions in T6-T7 and intervertebral disc associated with the presence of an extradural collection. 
(Fig. 2), where post-gadolinium T1-weighted MRI demonstrated a strong enhancement associated with the presence of an extradural collection (Fig. 3). These radiological findings were consistent with spondylodiscitis. Once blood cultures grow Bacteroides fragilis, the diagnosis of spondylodiscitis caused by Bacteroides fragilis was made. As expected on the basis of clinical course, Bacteroides fragilis strain was resistant to quinolones, while it was sensitive to metronidazole and piperacillin/tazobactam. A combined treatment with these drugs was started, while levoxacine was discontinued, with complete remission of fever. After 2 months follow-up, a thoracic spine MRI showed a complete regression of the inflammatory pattern.

\section{Discussion}

Spondylodiscitis due to Bacteroides fragilis in an uncommon finding [1]. Most cases of spondylodiscitis caused by Bacteroides fragilis result from hematogenous spread from a digestive portal of entry [2]. The patient's previous vertebral trauma might have represented the so-called "locus minoris resistentiae" [3] facilitating the initiation of a new focus of infection for Bacteroides fragilis. We assumed that the traumatized bone and discal tissues might be more vulnerable to invasive hematogenous bacterial infection, and that appendicular tract represented primary infective focus. Recently has been reported a case of spondylodiscitis caused by direct inoculation of Bacteroides fragilis that occurred after a percutaneous epidural adhesiolysis [4].

To the best of our knowledge, there are no previous reports of spondylodiscitis due to Bacteroides fragilis that occurred after a spine non-iatrogenic trauma.

As described in other clinical reports [5], our report confirms that PET/CT could be very useful in detecting spondy- lodiscitis in asymptomatic subjects.

\section{Conclusions}

Our case report suggests that spondylodiscitis due to Bacteroides fragilis should be considered in differential diagnosis in patients with fever of unknown origin and past history of spinal trauma, even if asymptomatic for spinal pain, especially in those who have experienced a recent abdominal infection.

\section{References}

1. Kawakami T, Suzuki H, Suzuki M, Hirose Y. Spondylodiscitis complicated by an epidural abscess and meningitis caused by Bacteroides fragilis. Intern Med. 2012;51(22):3189-3191.

2. Lechiche $\mathrm{C}$, Le Moing V, Marchandin H, Chanques G, Atoui N, Reynes J. Spondylodiscitis due to Bacteroides fragilis: two cases and review. Scand J Infect Dis. 2006;38(3):229-231.

3. Chan ED, Kong PM, Fennelly K, Dwyer AP, Iseman MD. Vertebral osteomyelitis due to infection with nontuberculous Mycobacterium species after blunt trauma to the back: 3 examples of the principle of locus minoris resistentiae. Clin Infect Dis. 2001;32(10):1506-1510.

4. Han IH, Choi BK, Nam KH, Kim SY. Bacteroides fragilis vertebral osteomyelitis complicated by percutaneous epidural adhesiolysis. Spine (Phila Pa 1976). 2013;38(7):E431-435.

5. Davison JM, Graham JA, Nieves NM, Yoest SM. PETCT confirms the diagnosis of pyogenic spondylodiscitis complicated by psoas abscess. BMJ Case Rep. 2013;2013. 